\title{
Uptake of neoadjuvant chemotherapy for invasive bladder cancer
}

\author{
Wassim Kassouf, MD, CM, FRSCS
}

Program Director and Associate Professor, McGill University Health Centre, Montreal, QC

Cite as: Can Urol Assoc J 2014;8(3-4):e294-5. http://dx.doi.org/10.5489/cuaj.2077 Published online April 14, 2014.

$\mathrm{R}$ adical cystectomy with pelvic lymphadenectomy remains the gold standard for muscle invasive bladder cancer (MIBC). ${ }^{1}$ However, survival rates are poor for patients treated with surgery alone ranging from $50 \%$ to $60 \%$ at 5 years. Impact of neoadjuvant MVAC (methotrexate, vinblastine, doxorubicin and displatin) in MIBC has been demonstrated by level 1 evidence. ${ }^{2}$ Meta-analysis of randomized prospective trials confirmed the $5 \%$ absolute improvement in survival when patients received neoadjuvant chemotherapy (NAC). ${ }^{3}$ Unfortunately, in Canada (and on a global level), NAC remains underutilized. In a Canadian multicentre study by Yafi and colleagues, of the 2287 patients who were treated with radical cystectomy at academic centres from 1998 to 2008, only 3.1\% received NAC and its use slightly increased to only $7 \%$ after $2005 .{ }^{4}$ I read the study by El-Gehani and colleagues with great interest. It is commendable to note such high rates of NAC $(57 \%)$ within the Alberta Urology Institute Database attesting to an effective collaboration among the disciplines and a strong buy-in to this approach from urologists and medical oncologists. ${ }^{5}$

\section{Should all patients with MIBC receive NAC?}

While NAC can be offered to all patients with MIBC, some centres, including the M.D. Anderson Cancer Center and the McGill University Health Centre, have adopted a risk-adapted approach. Although the NAC option is discussed with all eligible patients with MIBC, our institution has "strongly pushed" it primarily on patients with high-risk features: those with hydronephrosis, palpable mass on exam under anesthesia, invasion into adjacent organs (prostatic stroma or vaginal wall), lymphovascular invasion or on patients with concomitant variant histology, such as small cell differentiation or micropapillary features. Some of these variables were prognostic in the "pre- versus post" prospective randomized MVAC trial from the M.D. Anderson Cancer Center. ${ }^{6}$

While acknowledging the clinical-pathologic staging discrepancy within invasive bladder cancer, integrating a risk-adapted approach may avoid overtreatment in patients without high-risk features and where the absolute survival benefit with NAC is less. A recent study from the University of Southern California and the M.D. Anderson Cancer Center have provided reassurance with this approach; patients with CT2 and no high-risk features treated with upfront radical cystectomy demonstrated excellent 5-year cancer-specific survival of $83.5 \%$ despite a significant rate of pathologic upstaging on cystectomy. ${ }^{7}$ Taking the above information into consideration, the above-mentioned lower risk patients are more often treated with upfront radical cystectomy at our centre.

It is also important to note that the benefit of NAC in phase III trials have only been established in patients with cisplatin-based combination regimens. Patients who are cisplatin-ineligible due to compromised renal function should be treated with upfront radical cystectomy or a clinical trial if available. While the traditional cutoff for cisplatin eligibility has been an estimated glomerular filtration rate (eGFR) $>60 \mathrm{~mL} / \mathrm{min}$, some centres administer cisplatin-based chemotherapy in patients with eGFR between 50 and $60 \mathrm{~mL} /$ min using cisplatin split-dosing regimens.

Less controversial cases where NAC is not advocated are in patients with severe comorbidities, poor performance status, or highly symptomatic disease. Lastly, I have personally shied away from NAC in octogenarians and nanogenarians due to concern for potentially added morbidity in such a frail population.

\section{What NC regimen should be used?}

Gemcitabine plus cisplatin (GC) has been commonly used in the neoadjuvant setting, as in the study by El-Gehani and colleagues, ${ }^{5}$ despite no level 1 evidence supporting its bene- 
fit. When comparing MVAC and GC head-to-head in a noninferiority trial, von der Maase and colleagues showed similar median overall survival (14 vs. 15.2 months, $p=0.66$ ) and 5 -year overall survival $(13 \%$ vs. $15.3 \%, p=0.53)$ in patients with metastatic bladder cancer. ${ }^{8}$ Since GC was associated with significantly less toxicity, there has been an active push towards substituting MVAC with GC, even in the neoadjuvant setting. However, it remains unclear whether GC efficacy is similar to MVAC in the non-metastatic setting where the objective is cure rather than palliation. Since the NAC level 1 data are with MVAC, at our institution we have adopted high-dose MVAC where it can be administered in shorter 2-week cycles (rather than 4 weeks) and have been shown in a randomized trial to be associated with improved 5 -year overall survival $(21.8 \%$ vs. $13.5 \%$, $p=0.042$ ) and less toxicity compared to standard MVAC. ${ }^{9}$ Until this issue is resolved, we prefer that patients with MIBC continue to be treated with neoadjuvant high-dose MVAC, as the data is more robust in this setting and extrapolation on the efficacy of GC in the neoadjuvant setting remains to be defined. This concern has been supported within the colon cancer literature where irinotecan affected outcome in the metastatic setting, but did not demonstrate a benefit in the perioperative setting.

\section{Conclusion}

As one can quickly remark, the underutilization of NAC with MIBC is only the tip of the iceberg when evaluating how the medical community manages bladder cancer patients at large. Of greater concern is that most (55\%) patients with MIBC who are over 70 never receive any form of definitive therapy..$^{10}$ There are many other nuances and variations in the care of bladder cancer patients across the different stages that need to be addressed and standardized. We are currently working on a national, quality of care bladder cancer meeting that will be held in the fall of 2014. A mix of urologists, medical oncologists, radiation oncologists, pathologists, ostomy nurses, and patients will meet over 2 days to develop standards and provide consensus of how patients with bladder cancer should be managed across Canada. This much-needed event will be generously supported by Bladder Cancer Canada, in partnership with the Canadian UroOncology Group and the Canadian Urological Association.

Competing interests: Dr. Kassouf is an Advisory Board member and a speaker for Amgen and Astellas. He has also received grants and honoraria from these companies. He is currently participating in unpaid clinical trials.

\section{References}

1. Stenzl A, Cowan NC, De Santis M, et al. European Association of Urology (EAU). Treatment of muscleinvasive and metastatic bladder cancer: update of the EAU guidelines. Eur Urol 2011;59:1009-18. http:// dx.doi.org/10.1016/i.eururo.2011.03.023

2. Grossman $H B$, Natale RB, Tangen $C M$, et al. Neoadjuvant chemotherapy plus cystectomy compared with cystectomy alone for locally advanced bladder cancer. N Engl J Med 2003;349:859-66. http://dx.doi. org/10.1056/NEJMoa022148

3. Advanced Bladder Cancer (ABC) Meta-analysis Collaboration. Neoadjuvant chemotherapy in invasive bladder cancer: Update of a systematic review and meta-analysis of individual patient data advanced bladder cancer (ABC) meta-analysis collaboration. Eur Urol 2005;48:202-5. http://dx.doi.org/10.1016/i. eururo.2005.04.006

4. Yafi FA, Aprikian AG, Chin JL, et al. Contemporary outcomes of 2287 patients with bladder cancer who were treated with radical cystectomy: A Canadian multicentre experience. BJU Int 2011;108:539-45. http://dx.doi.org/10.1111/i.1464-410X.2010.09912.x

5. El-Gehani F, North $S$, Ghosh $S$, et al. Improving the outcome of patients with muscle invasive urothelial carcinoma of the bladder with neoadjuvant gemcitabine/cisplatin chemotherapy: A single institution experience. Can Urol Assoc J 2014;8:e223-9. http://dx.doi.org/10.5489/cuaj.1643

6. Millikan R, Dinney C, Swanson D, et al. Integrated therapy for locally advanced bladder cancer: final report of a randomized trial of cystectomy plus adjuvant M-VAC versus cystectomy with both preoperative and postoperative M-VAC. J Clin Oncol 2001;19:4005-13.

7. Culp SH, Dickstein RJ, Grossman HB, et al. Refining patient selection for neoadjuvant chemotherapy before radical cystectomy. J Urol 2014;191:40-7. http://dx.doi.org/10.1016/i.juro.2013.07.061

8. von der Maase H, Sengelov L, Roberts JT, et al. Long-term survival results of a randomized trial comparing gemcitabine plus cisplatin, with methotrexate, vinblastine, doxorubicin, plus cisplatin in patients with bladder cancer. J Clin Oncol 2005;23:4602-8. http://dx.doi.org/10.1200/JC0.2005.07.757

9. Sternberg CN, de Mulder PH, Schornagel JH, et al. Randomized phase III trial of high-dose-intensity methotrexate, vinblastine, doxorubicin, and cisplatin (MVAC) chemotherapy and recombinant human granulocyte colony-stimulating factor versus classic MVAC in advanced urothelial tract tumors: European Organization for Research and Treatment of Cancer Protocol no. 30924. J Clin Oncol 2001;15:2638-46.

10. Gray PJ, Fedewa SA, Shipley WU, et al. Use of potentially curative therapies for muscle-invasive bladder cancer in the United States: Results from the National Cancer Data Base. Eur Urol 2013;63:823-9. http://dx.doi.org/10.1016/i.eururo.2012.11.015

Correspondence : Dr. Wassim Kassouf, Division of Urology, McGill University Health Centre, 1650 Cedar Ave., Rm 18-315, Montreal, QC H3G 1A4; fax: 514-934-8297; wassim.kassouf@muhc.mcgill.ca 